\title{
Collective Behavior in a Local Causal Model of Quantum Theory
}

\author{
Hans H. Diel \\ Diel Software Beratung und Entwicklung, Sindelfingen, Germany \\ Email: diel@netic.de
}

How to cite this paper: Diel, H.H. (2017) Collective Behavior in a Local Causal Model of Quantum Theory. Open Access Library Journal, 4: e3898.

https://doi.org/10.4236/oalib.1103898

Received: August 21, 2017

Accepted: September 12, 2017

Published: September 15, 2017

Copyright (c) 2017 by author and Open Access Library Inc.

This work is licensed under the Creative Commons Attribution International License (CC BY 4.0).

http://creativecommons.org/licenses/by/4.0/

\begin{abstract}
The non-localities in quantum theory (QT) (the most famous example is expressed in the violation of Bell's inequality in experiments) impede the construction of a local causal model of QT including quantum field theory (QFT). The laws of collective behavior may be considered to be types of non-local laws: laws that apply to the collection of system components as a whole. The article presents a proposal for the treatment of the non-localities that exist in QT/QFT by the concepts of collective behavior. The basic components of the collective behavior are the spatial elements of the causal model of QT/QFT proposed by the author.
\end{abstract}

\section{Subject Areas}

Quantum Mechanics, Theoretical Physics

\section{Keywords}

Causal Models, Collective Behavior, Quantum Field Theory, Lattice Gauge Theory, Decoherence, Spacetime Models

\section{Introduction}

The work described in this paper had two triggers. The initial trigger was the authors' attempt to develop a comprehensive computer model of quantum theory (QT) that enables the simulation of an as large as possible set of QT (Gedanken-) experiments. Soon it was realized that the development of a computer model for a particular area of physics requires the availability of (or feasibility of constructing) a causal model of that area of physics. The second trigger was the pursuit of the EPR-experiment and of Bell's inequality. The refutation of Bell's inequality (see [1]) in experiments such as the Aspects experiment (see [2]) is 
interpreted by physicists as an indication (or proof) that local causal models of QT are not possible. An analysis of the subject causal model requires, first of all, a clear understanding of a local causal model. In Section 2, a formal definition of a causal model is given that provides a suitable base for the subsequent discussion. ${ }^{1}$

The definitions given in Section 2 include the definition of a local causal model. However, it turned out that the subject local causal model, or more generally, the subject locality in theories of physics, is too complex to analyze in terms of a single "strong" type of locality that in this paper is called "space-point locality". It is concluded that different degrees of locality should be distinguished to enable a reasonable discussion. "Object-locality" is introduced to enable the discussion about what kind of locality is desirable when space-point locality cannot be generally provided for a specific theory of physics. In Section 2.3, the roles of global laws of theories of physics are analyzed.

An important type of non-local laws that may determine the causal dynamics of a system are the rules and laws that determine the collective behavior of a system that consists of a large number of components of the same type. Physicists detected that the behaviors of collections of a large number components follow special laws and that the application of these laws to specific areas of physics may lead to an improved understanding of the related theories. Because the laws of collective behavior apply to the collection of components as a whole, they are included in Section 2.3 as types of non-local laws of physics.

In Sections 3 and 4, the concepts and techniques of collective behavior are applied to the development of a refinement of a proposed (local) causal model of QT, including quantum field theory (QFT). This demonstrates the potential value of the concepts and techniques of collective behavior for the development of an improved understanding of still weak areas of physics.

The need for Causal Models: The author claims that causal models are important for the following reasons:

a) From the appearance of the first theories of physics until today, the determination of causal relationships, including the prediction of the results of physical experiments, has been the primary goal for doing physics.

b) Our inability to specify detailed causal relationships in certain areas of physics may be tolerated for some time, but should not lead to the explicit negation of causality or to making allowances for the lack of a better understanding. There are different areas of physics where this argument is considered (by the author) to apply to differing degrees: 1) In statistical mechanics and thermodynamics, the causal relationships are understood, although not computable at a detailed level; 2) The assumption of indeterminism in QT is a good working assumption, but declaring the indeterminism of QT as an inherent feature of QT (that could even be proven mathematically) is at least premature; 3) Not recognizing the measurement problem of QT as something for which a suitable solu${ }^{1}$ Note that a causal model is not necessarily (realized by) a computer model. A causal model is still a "physical model". 
tion is highly desirable (or even necessary) is incomprehensible to the author.

c) The goal of the construction of a causal model limits the set of possible theories and models; however, this limitation may lead to models that are more realistic.

The need for Local Causal Models: The conclusion drawn from the violation of Bell's inequality in experiments, that local causal models of quantum theory apparently are not feasible, was somewhat of a shock to many physicists. It was considered as the refutation of what most physicists believe that causal effects can propagate to distant locations only with final speed through a series of propagations through neighbor locations. Instead, the instantaneous propagation of effects to distant locations, which Einstein called "spooky actions at a distance", appeared to be possible. There are probably not many physicists who are not concerned about the possibility of "spooky actions at a distance". While it does not appear to be reasonable to plainly reject the possibility of non-local causal action propagation, nor to assume the existence of non-local causal action propagation in general, the author claims that it is necessary to look for causal models in identified situations where, instead of the "space-point locality", a weaker degree of locality may occur. The weaker type of locality needs to be defined in the context of a causal model. Within this article, two possible directions are outlined for the definition of locality weaker than space-point-locality: 1) the “object-locality" and 2) "collective-system-locality" described in Section 2.3.

\section{Formal Definition of a Causal Model}

The specification of a causal model of a theory of physics consists of: 1) the specification of the system state; 2) the specification of the laws of physics that define the possible state transitions when applied to the system state; and 3) the assumption of a "physics engine".

The physics engine represents the overall causal semantics of causal models. It acts upon the state of the physical system. The physics engine continuously determines new states in uniform time steps. For the formal definition of a causal model of a physical theory, the continuous repeated invocation of the physics engine is assumed to realize the progression of the state of the system.

Physics engine $(S, \Delta t):=\{$

DO UNTIL(nonContinueState $(S))\{$

$S \leftarrow$ applyLawsOfPhysics $(S, \Delta t) ;$

\}

\}

The system state defines the components, objects and parameters of the theory of physics that may be referenced and manipulated by the causal model. In contrast to the physics engine, the structure and contents of the system state is specific for the causal model being specified. Therefore, the following is just an example of a possible system state specification.

Systemstate: $=\{$ spacepoint... $\}$ 
Spacepoint: $=\left\{x_{1}, x_{2}, x_{3}, \psi\right\}$

$\psi:=\left\{\right.$ stateParameter $_{1}, \ldots$, stateParameter $\}$

The laws of physics: The refinement of the statement $S=$ apply Laws Of Physics $(S, \Delta t)$; defines how an "in" state s evolves into an "out" state $s$.

$$
\begin{gathered}
L_{1}:=\operatorname{IF} c_{1}(s) \text { THEN } s \leftarrow f_{1}(s) ; \\
L_{2}:=\operatorname{IF} c_{2}(s) \text { THEN } s \leftarrow f_{2}(s) ; \\
\quad \vdots \\
L_{n}:=\operatorname{IF} c_{n}(s) \text { THEN } s \leftarrow f_{n}(s) ;
\end{gathered}
$$

The "in" conditions $c_{i}(s)$ specify the applicability of the state transition function $f_{i}(s)$ in basic formal (e.g., mathematical) terms or refer to complex conditions that then have to be refined within the formal definition.

The state transition function $f_{i}(s)$ specifies the update of state $s$ in basic formal (e.g., mathematical) terms or refers to complex functions that then have to be refined within the formal definition.

To enable non-deterministic theories ("causal" does not imply deterministic) an elementary function

RANDOM (value range, probability distribution) may also be used for the specification of a state transition function.

The set of laws $L_{1}, \cdots, L_{n}$ has to be complete, consistent and reality conformal (see [3] for more details).

In addition to the above described basic forms of specification of the laws of physics by $L_{n}:=\operatorname{IF} c_{n}(s)$ THEN $s \leftarrow f_{n}(s)$, other forms are also imaginable and sometimes used in this article. ${ }^{2}$

Note the following comments on the notation used for the specification of causal models. Although in mathematics and in programming languages the " $=$ " sign is used for three different purposes, in this article three different notations are used for the different purposes:

1) “=” indicates a relation, as in $\mathrm{a}=\mathrm{b}$, reading a equals $\mathrm{b}$.

2) ":=" means "is defined as". For example, spacepoint: $=\left\{x_{1}, x_{2}, x_{3}, \psi\right\}$ means spacepoint is defined as....

3) " $\leftarrow$ " indicates a value assignment. $x \leftarrow y$ means that the value of the expression on the right-hand side $(y)$ is assigned to the item on the left-hand side (x).

This notational distinction is used only in causal-model specifications. In traditional mathematical expressions occurring in this paper, the traditional meaning of the "=" sign is used. The distinction of the three types of "equality" has significant implications for the specification of a causal model. It means that typical mathematical equations, such as $T=\frac{1}{2} m \dot{x}^{2}$ (see below), must not be taken unchanged for the laws of physics $L_{i}$. The traditional symmetric equal sign appearing in " $T=\frac{1}{2} m \dot{x}^{2}$ ” must be replaced by the asymmetric “ $\leftarrow$ ". As a

${ }^{2}$ This article does not contain a proper definition of the used causal model specification language. The language used is assumed to be largely self-explanatory. 
consequence, $T \leftarrow \cdots$ and $\dot{x} \leftarrow$ cannot both occur in a causal evolution step (or even within the whole causal model).

Example 1-A causal model: Many areas of physics can be described by starting with a specific Lagrangian. For a description of the causal relationships, i.e., the evolution of the system state, the equation of motion is the major law. The equation of motion can be derived from the Lagrangian by using the Euler-Lagrange equation.

The Lagrangian for classical mechanics is

$L=V-T$ with

$$
V=V(x), T=\frac{1}{2} m \dot{x}^{2} .
$$

The Euler-Lagrange equation leads to the equation of motion

$$
m \ddot{x}=\frac{\delta V}{\delta x} .
$$

The specification of the laws of classical mechanics can be given by a list $\left(L_{1}, \cdots, L_{n}\right)$ that distinguishes different cases or by a single general law. The single general law is

$L_{1}:=$ IF (TRUE) THEN FOR (all Particles $\left.P_{i}\right)\{$

$P_{i} \leftarrow$ applyEquationOfMotion $\left.\left(P_{i}\right) ;\right\}$

Thus, the system state has to contain

systemstate: $=\{$

space;

particles: $=P_{1}, \cdots, P_{n}$;

field $V:=V(x)$;

Particle $P:=\{m, x, \ddot{x}, \dot{x}\}$

\}

\subsection{Types and Properties of Causal Models}

Spatial causal model: A causal model of a theory of physics is called a spatial causal model if (1) the system state contains a component which represents a space, and (2) all other components of the system state can be mapped to the space.

There exist numerous textbooks on physics (mostly in the context of Relativity theory) and on mathematics which define the essential features of a "space". For the purpose of the present article a more detailed discussion is not required. For the purpose of this article and the subject locality it is sufficient to request that the space (assumed with a spatial model) supports the notions of position, coordinates, distance, and neighborhood.

Example 2-A spatial causal model: A possible type of a spatial causal model is the cellular automaton (CA). The classical CA consists of a k-dimensional grid of cells. The state of the CA is given by the totality of the states of the individual cells.

$$
\text { State: }=\left\{s_{1}, \cdots, s_{n}\right\}
$$


With traditional standard CAs, the cell states uniformly consist of the same state components

$$
s_{i}:=\left\{s_{i}^{1}, \cdots, s_{i}^{j}\right\}
$$

Typically, the number of state components, $j$, is 1 , and the possible values are restricted to integer numbers. The dynamical evolution of the CA is given by the "update-function", which computes the new state of a cell and of the neighbor cells as a function of the current cell state.

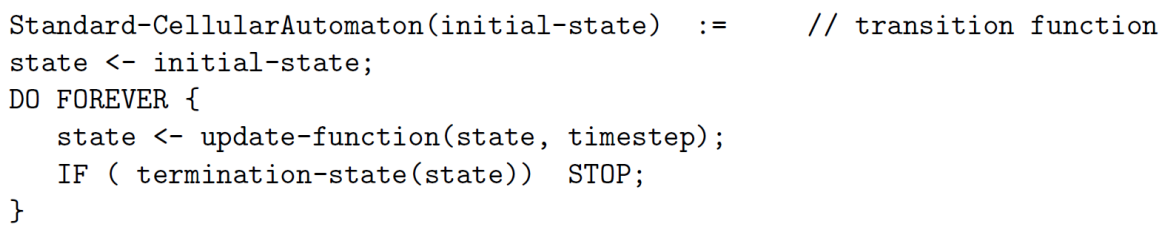

The full functionality and complexity of a particular CA is concentrated in the update-function. As Wolfram (see [4]) and others (see, e.g., [5]) have shown, a large variety of process types (e.g., stable, chaotic, pseudo-random, and oscillating) can be achieved with relatively simple update-functions.

\subsection{Formal Definition of a Local Causal Model}

The definition of a local causal model presupposes a spatially causal model (see above). A (spatially) causal model is understood to be a local model if changes in the state of the system depend on the local state only and affect the local state only. The local state changes can propagate to neighboring locations. The propagation of the state changes to distant locations; however, they must always be accomplished through a series of state changes to neighboring locations. ${ }^{3}$

Based on a formal model definition of a causal model, a formal definition of locality can be given. We are given a physical theory and a related spatially causal model with position coordinates $\mathrm{x}$ and position neighborhood $\mathrm{dx}$ (or $x \pm \Delta x$ in case of discrete space-points).

A causal model is called a local causal model if each of the laws $L_{i}$ applies to no more than a single position $\mathrm{x}$ and/or to the neighborhood of this position $x \pm d x$.

In the simplest case, this arrangement means that $L_{i}$ has the form

$$
L_{i}: \operatorname{IF} c_{i}(s(x)) \text { THEN } s^{\prime}(x)=f_{i}(s(x)) ;
$$

The position reference can be explicit (for example, with the above simple case example) or implicit by reference to a state component that has a well-defined position in space. References to the complete space of a spatially extended object are considered to violate locality. References to specific properties of spatially extended objects do not violate locality. ${ }^{4}$

${ }^{3}$ Special relativity requests that the series of state changes does not occur with a speed which is faster than the speed of light. This requirement is not considered within the present article. ${ }^{4}$ With the distinction of "space-point locality" and "object-locality" (see below) a refinement of this rule is appropriate. 
Note: In addition to space-point related locality, it is also possible to consider "time locality". The above given definition of a causal model implies time locality insofar as it does not allow that the physics engine and the laws of physics (included in the causal model) refer to system states at different time slices.

\subsection{Non-Local Laws of Physics}

Section 2.2 presented formal conditions for the specification of a local causal model of a theory or area of physics. It is reasonable to analyze the physical conditions that may impede or prevent the construction of a local causal model. The major type of obstacle for the construction of a local causal model of a theory of physics is if the theory contains laws or principles that are non-local by nature. In the following, three types of non-local laws of physics are discussed: 1) global laws of physics; 2) object-local laws of physics; and 3) collective behavior laws of physics.

Global laws of physics: There are numerous laws of physics (in all areas of physics) that are assumed to apply to a complete (closed) system or even to the universe as a whole. Some famous examples are

- the conservation laws (energy conservation, momentum conservation;

- the entropy law (the second law of thermodynamics);

- mass-energy conversion $E=m c^{2}$.

If a global law is included in the formal specification of the causal model, the law representation $L_{i}$ in the causal model must refer to the complete system state, and this would violate the conditions for a local causal model. However, there is a way out that enables the provision of local causal models to include global laws. Most of the global laws of physics can be and have been mapped to a local situation. For example, the laws of energy conservation and momentum conservation are reflected in the symmetry requirements on the Lagrangian of the pertinent theories. The local derivative of the global law is suitable for a local causal model. With some global laws, explicit inclusion in the list of laws of the causal model is not necessary because (1) the law is already implicitly reflected in the other laws (example: constant c) or (2) the law does not affect the causal progression of the system state (example: entropy law). Some global laws are already implemented by the definition of the "physics engine". In other words, the definition of the physics engine itself implies certain types of "meta-laws." Examples of global laws implied by the physics engine are the assumptions of time symmetry and space symmetry. Because of Noether's theorem, time symmetry implies the global law of energy conservation, and space symmetry implies momentum conservation. ${ }^{5}$ In summary, the mapping of the global laws of physics to a local causal model does not in general pose a problem.

Object-related laws of physics: Many areas of physics address compound objects that occupy a larger area of space. In the classical areas of physics, the provision of a local causal model that includes compound objects does not

${ }^{5}$ If the symmetries are not violated by the local laws (e.g., by the Lagrangian). 
represent a problem because the combined causal effect of the spatially distributed pieces of the object is typically a simple aggregation. In addition, because of the final speeds of the propagation of actions, it is ensured that causality and locality is preserved. This is different in quantum physics. The EPR experiment and the violation of Bell's inequality raised doubts about the feasibility of local causal models of QT. In [6] and [7], further areas of QT are shown in which the development of a local causal model apparently is not possible if locality is understood as space-point locality. Instead, in [6], "object-locality" is introduced and "quantum objects" are introduced as elementary units of causality and locality. This means that the violation of space-point-locality in QT/QFT is confined to quantum objects. In Section 5, a proposal is described for the explanation of part of the non-localities of QT/QFT in terms of collective behavior.

Laws of physics of collective behavior: Other types of non-local laws that may determine the causal dynamics of a system are the rules and laws that determine the collective behavior of a system that consists of a large number of components. Because the laws of collective behavior apply to the collection of components as a whole, they are considered a type of non-local laws of physics. Collective behavior is discussed in more detail in the following sections.

\section{Collective Behavior}

Collective behavior systems are understood as systems consisting of a large number of components of the same type. Collective behavior systems have been studied by physicists for more than 150 years. Some physicists (e.g., [8]) consider L. Boltzmann as the physicist who established the basis of theories of collective behavior with his work on thermodynamics. In particular, the second law of thermodynamics (entropy law) may be viewed as describing collective behavior systems phenomena. Other work that is usually mentioned in the literature as early influential work is the Ising model (see [9]), which is a model of the development of an initially unmagnetized collection of atoms into a system with strong magnetization. More recent work in which the "collective behavior laws" have been applied to quantum theory are associated with the name K. Wilson et al. (see [10]) on lattice gauge theory (LGT) (see [11]). Physicists who work in areas where collective behavior has been recognized believe that general laws of collective behavior exist: laws that cannot be derived from the corresponding single component systems, nor is it possible to reduce these laws to laws for the single component system. ${ }^{6}$

The research of physicists for general laws for collective behavior systems resulted in findings that influenced some theories of physics (e.g., quantum chromodynamics) considerably.

Major characteristics of collective behavior: It is not possible in this article to provide an introduction to collective behavior systems. Nevertheless, it is necessary to list at least some major characteristics of collective behavior systems:

${ }^{6}$ This is sometimes interpreted as the end of reductionism. 
- The collective behavior is mainly caused by the continuous interactions among the many components of the system. The frequency, scope and other properties of the interactions depend on a number of parameters such as

- the type of interaction force;

- the reach of interaction force;

- the density of the system components within the volume covered by the system, i.e., the spatial distance between the interacting components, and the energy (distribution) of the components. A special dynamically varying form of energy distribution that is often part of models of collective behavior is fluctuations. In addition to the energy parameter, fluctuations occur with additional parameters of the system components;

- the spatial distribution of the interacting components. With some models of collective behavior, the spatial distribution is simplified and takes the form of a lattice. With other models the spatial distribution evolves through a process called percolation.

- Depending on the systems energy, different phases of the systems behavior can be observed often. Phase transitions typically occur within sharp energy ranges. Another phenomenon often associated with energy decrease is symmetry breaking.

- Models for the treatment of collective behavior systems typically assume discrete space-time, for example, lattices. ${ }^{7}$

- Collective behavior is always statistical behavior. Therefore, the laws of statistical mechanics are generally applied in analyses of the various types of collective behavior.

\section{(Causal) Models of Collective Behavior}

It is possible to demonstrate specific aspects of collective behavior in form of a model. In physics, such models typically express some causal relationship as well. The models may be described in a form similar to the formal specification of a causal model given in Section 2. Whether this means that collective behavior can/should be part of the causal model of a theory of physics will be discussed following the examples. Three examples of models of specific collective behavior are used as the basis for the discussion.

Example 3-Entropy model: The model shows the change of the entropy of a system consisting of $\mathrm{n}$ components and a given initial-state. To obtain a specific behavior that adheres to the entropy law, the function count-nonequivalentstates() and the state change caused by perform-interaction() would have to be specified more concretely.

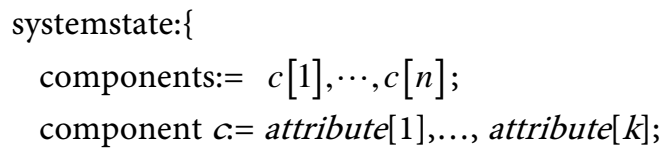

${ }^{7}$ With existing models such as the Ising model and lattice gauge theory, the assumption of discrete space-time is merely a technique to ease the calculations. With the causal model of QT/QFT described in Section 4, the assumption of discrete space-time has physical implications. 


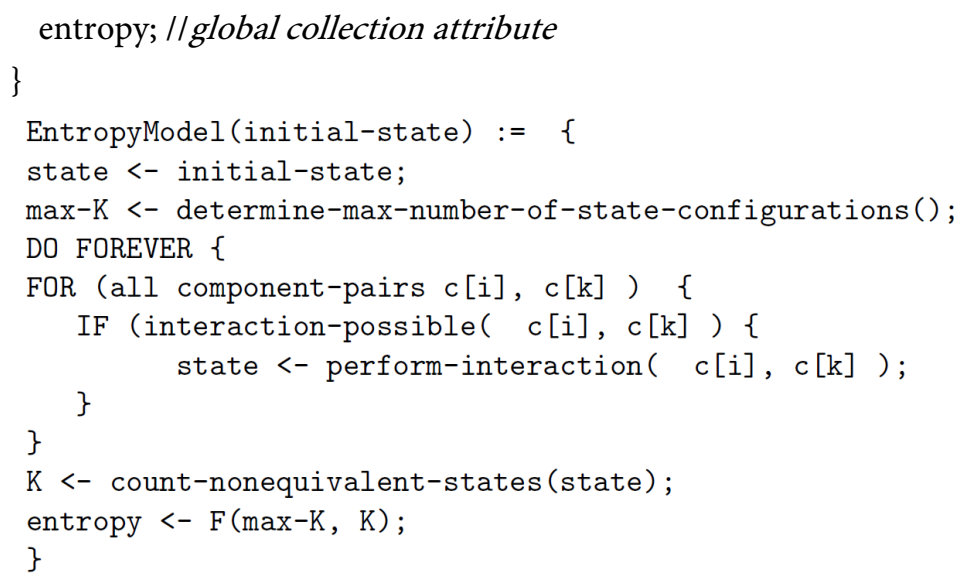

The example model shown violates a number of formal criteria for a causal model as described in Section 2. The violations, however, are consequences of the different purposes of models of collective behavior.

- The entropy model contains as part of the system state a component "entropy". The state component entropy, in contrast to ordinary state components, has no relevance for the causal evolution. It never appears as an input parameter for the update-state laws. Entropy is a global system state attribute, which means it would disable a local causal model.

- The model (as shown above) contains several undefined functions that in a complete formal causal model would have to be specified. Part of the unspecified functions have been omitted to keep this article reasonably compact. However, there are also functions (e.g., "state $\leftarrow$ perform-interaction $(c[i]$, $\mathrm{c}[\mathrm{k}])^{\text {") }}$ that can be properly provided only when the model is applied to a concrete area of physics such as thermodynamics. This means that the model shown above is an "abstract model" that demonstrates that the concept of entropy can be applied to various areas of physics.

- The model is a non-local model because the two spatially separated components $c[\mathrm{i}]$ and $c[\mathrm{k}]$ occur as input and output parameters of the state update function.

Example 4-Ising model: Based on a collection of components (e.g., atoms) residing in a lattice, with possible states +1 or -1 , the Ising model demonstrates increasing magnetization as a function of decreasing temperature. The model also shows the symmetry breaking when the magnetization becomes non-zero. The Hamiltonian $H$ of the system is typically specified as

$$
H=\frac{1}{2} \sum_{i, j} J_{i, j} s_{i} \cdot s_{j}-M \cdot \sum_{i} s_{i}
$$

where $J_{i, j}$ is the coupling constant and $M$ is the magnetic field.

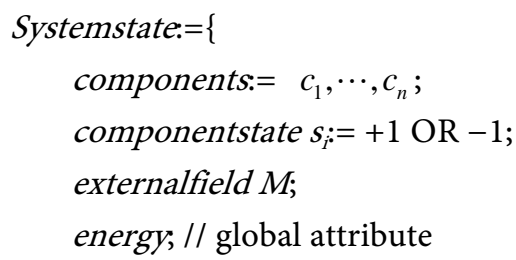




\section{\}}

Ising-Model(initial-state): $=\{$

state $\leftarrow$ initial-state;

DO FOREVER \{

FOR (all component-pairs $c_{i}$, neighbor $\left.\left.\left(c_{i}\right) c_{j}\right)\right)\{$

$H \leftarrow \frac{1}{2} \sum_{i, j} J_{i, j} s_{i} \cdot s_{j}-M \cdot \sum_{i} s_{i}$

\}

\})

Remarks similar to those given above for Example 3 can be made for Example 4: (1) redundant and non-local system state components, and (2) incompletely defined abstract functions. In contrast to Example 3, however, the Ising model is a local model because the interactions occur only between neighbor components.

Example 5-Percolation: Percolation is another typical example of collective behavior that can be observed with various processes in nature. A given area of space is gradually filled with an increasing number of components and/or with components that increase in size. When a certain occupancy of space by the components is reached, the behavior of the system changes radically, i.e., a phase transition occurs.

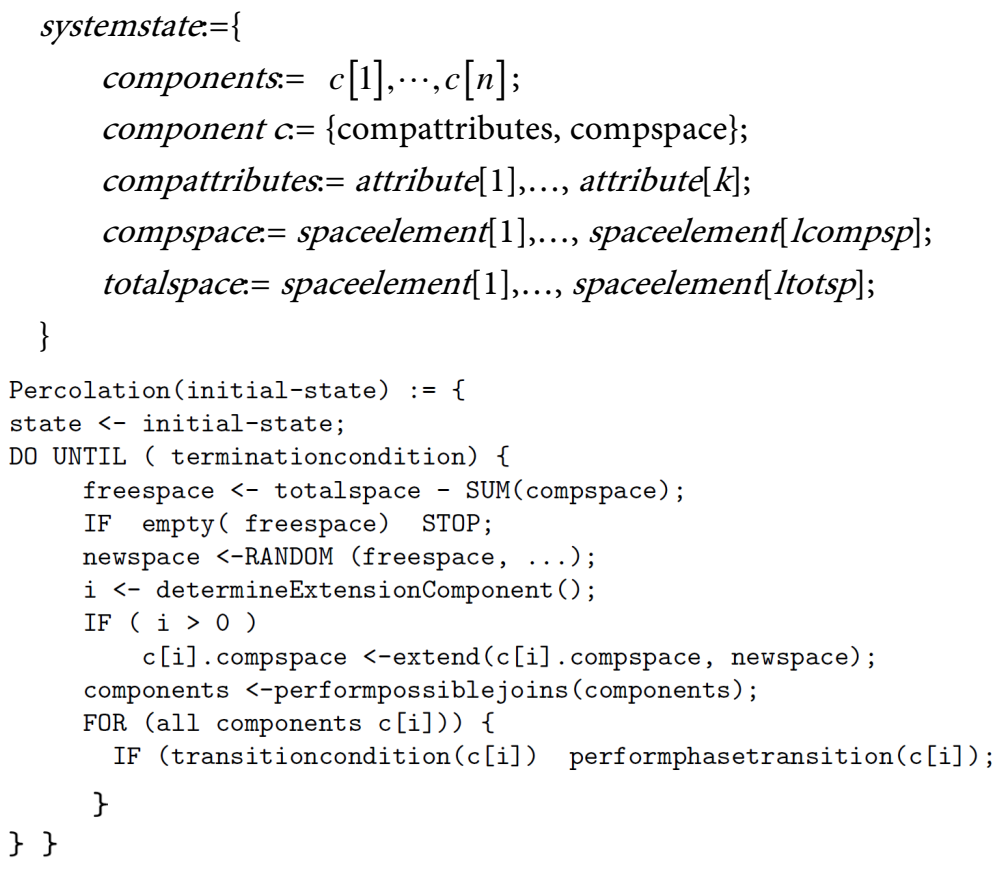

Example 5, Percolation, does not contain redundant global system state components. However, like Examples 3 and 4, the percolation example contains unspecified "abstract functions" (e.g., transition condition(c[i])) to enable its applicability to different specific areas.

In general, the broad range of applicability of the concepts and models of collective behavior is obtained by abstraction. A subset of the complete set of system state components and causal laws is carefully selected such that new, more 
general laws of collective behavior can be derived. To (1) establish a law of collective behavior or (2) verify that a given law of collective behavior is applicable to a concrete example, it must be verified that the concrete example is a valid instance of the abstract model of collective behavior. Typically, this can be verified only if the concrete example is understood in detail.

With entropy (Example 3), for example, the details of the interaction function ("state $\leftarrow$ perform-interaction $(\mathrm{c}[\mathrm{i}], \mathrm{c}[\mathrm{k}])$ ") must be understood to verify the applicability of the entropy law and the entropy model. For thermodynamics and the parameters energy and temperature, the verification is trivial because the entropy law was found by abstraction of well-understood details of thermodynamics, including details of how system components interact. Application of the entropy law to areas other than thermodynamics or parameters other than energy and temperature or other types of interactions is doubtful. This does not exclude that it may be reasonable to assume the general applicability of the entropy law as a conjecture (which may be verified in the future). It is even more doubtful to assume the general applicability of the entropy law to derive further laws.

\section{Collective Behavior in the Causal Model of QT/QFT}

To construct a comprehensive causal model of QT/QFT, the author had to propose some solution for QT/QFT "problem areas," namely, the QT measurement problem, the QT “interference collapse rule," entanglement and a causal model of QFT interactions (see [3]). The solutions chosen for the causal model are based on the proposed model of QFT interactions (see [6]). In addition, it had to be determined what kind of locality can be provided in the causal model if the strong spacepoint locality cannot be provided (as has been concluded from the violation of Bell's inequality by the EPR experiment). The provision of spacepoint locality was found to be a problem not only with entanglement but also with the subjects collapse of the wave function, the interference collapse rule, and the treatment of QFT interactions, i.e., (accidentally?) with all of the problem areas mentioned above. The solution chosen for the locality problem is the definition of "object-locality" and the identification of "quantum objects" as the elementary units of causality and locality.

In Section 2.3, three types of non-local laws of physics are distinguished: global laws, object local laws and collective behavior laws. For the author, this led to the question of whether the object locality assumed in the causal model of QT/QFT can be explained or refined by collective behavior. As will be described in the following, there are indeed model refinements possible by the application of certain concepts and techniques of collective behavior to the causal model of QT/QFT. The major commonalities that support the application of collective behavior concepts to the causal model of QT/QFT are as follows:

1) with both collective behavior systems and the causal model of QT/QFT, interactions play a key role, and

2) with both collective behavior systems and the causal model of QT/QFT, dis- 
creteness of space, time and the system components is an important assumption.

\subsection{Overall Approach}

The major area of refinement of the causal model of QT/QFT is an improved explanation of object-locality within quantum objects. The identification of quantum objects as the elementary units of (object-) locality and causality means that the laws of QT/QFT that specify the state transitions in the causal model may violate space-point locality as long as the set of referenced positions in space is confined to the space occupied by the quantum object. In the causal model, this non-spacepoint-locality within the quantum object typically has been implemented by the laws of causal dynamics referring to object-global components or parameters. The object-global components or parameters may be referenced or changed instantaneously as a whole.

The overall approach for the application of collective behavior concepts to the causal model of QT/QFT can be summarized as follows:

- The internal dynamics of quantum objects, including interactions between quantum objects, are described in terms of typical collective behavior processes. In particular, the instantaneous changes of object-global parameters are refined in terms of collective behavior processes with (instantaneous) phase transitions. The major types of collective behavior processes that occur in the refined model are as follows:

\section{a) Clustering/De-clustering}

Clustering of components to form larger objects, possibly with significantly different properties, has much in common with percolation (see Example 5). When the clustering reaches a certain size or a certain occupancy of the available space, an instantaneous change of behavior occurs. For the causal model of QT/QFT, declustering, the reverse phase transition, is also very important. The measurement process, the collapse of the wave function and the collapse of interference are explained by declustering.

b) Fluctuations

In this article, fluctuations are understood as the continuous separation of smaller parts of a larger object that (temporarily) reduces the object that generates the fluctuations. The virtual particles of QFT are assumed to be caused by fluctuations.

c) Interactions

Interactions between the various types of objects (particles, virtual particles, clusters of space elements) are the basis of most of the collective behavior (and of other phenomena). As described in Section 3 ("Major characteristics of collective behavior") the detailed causal effect of interactions depends on a number of parameters of the interacting components.

Clustering/declustering and fluctuation are closely related to the creation/annihilation operators of QFT.

- Collective behavior always applies to collections of components. In the causal model, the collective behavior components are called "space element clus- 
ters". Space element clusters may exist in different aggregate states and roles. One of the possible roles is comparable to virtual particles.

- The detailed dynamics of the space element clusters are highly interrelated with the structure and dynamics of space-time that is assumed for the causal model as described in [6]. A short overview is given below.

\subsection{Space-Time Structure in the Causal Model of QT/QFT}

In the causal model of a theory of physics (see Section 2), space and time are treated differently. Time and the progression of time is an inherent feature of the physics engine associated with each individual quantum object. Time is a quantum object-local property. In contrast, in the causal model, space is the global object that is referenced by quantum objects whenever global interrelationships among quantum objects must be implemented. The assumption/requirement that the space itself changes dynamically (e.g., expands and changes its curvature) resulted in the association of a separate physics engine to the space. The physics engine assigned to the (global) space determines the speed of changes (e.g., expansions and curvature changes) of the space.

Although the general causal model described in Section 2 shows a rather non-unified picture for space and time, the (specific) causal model of QT/QFT, including space-time considerations, however, leads to an integration of the two concepts such that compatibility with GRT and SRT is largely maintained. The (re-) integration of space and time in the causal model of QT/QFT is caused by the model assumption that all space dynamics (e.g., expansions and curvature changes) are triggered by events of the quantum objects (e.g., creation and movement of quantum objects) and the assumption that the events of the quantum objects occur according to the quantum objects local times.

The causal model uses the theory of causal dynamical triangulation (CDT) (see [12]) as one of its bases because CDT matches a number of features that are also supported by the causal model of QT/QFT, as described in [7]. CDT assumes triangles (two-dimensional) and tetrahedrons (three-dimensional) as discrete building blocks of space-time and offers a theory of the dynamical evolution of such a space-time. The use of CDT as the base for the space-time structure of the causal model of QFT/QT, however, resulted in the necessity for additional specifications. Primarily, a causal model of the dynamics of space (e.g., emergence of space, change of curvature) had to be specified. As the major deviation from standard CDT, in the causal model of QT/QFT, time is not a (fourth) dimension of the basic space (-time) elements. The integration of space and time is obtained by the fact that the evolution of space is caused by the evolution of the quantum objects and the evolution of the quantum objects occurs with the quantum objects local proper times.

In the causal model of QT/QFT, the following assumptions are made concerning space:

- The dynamics of space (i.e., creation and expansion of space, change of space curvature) evolves from local sources to the largest possible scope (i.e., to the 
whole universe).

- The space changes start at the elementary units of causality and locality, the quantum objects (see [7]). Large-scale changes and large-scale sources of changes are aggregations of changes triggered by quantum objects.

- Space changes propagate with limited speed, the speed of light.

- The space is an active object, i.e., its dynamics is driven by a physics engine with a global proper-time interval.

\subsection{Clustering and De-Clustering}

Starting with the basic elements of space, the triangulation simplexes, clusters of space elements emerge through processes that are a combination of fluctuations and clustering. The resulting objects are called "space element clusters" in this article. Space element clusters develop as the result of collective behavior and they are the source of further collective behavior. Depending on specific attributes and properties, different types of space element clusters can be distinguished as shown in Table 1.

The basic clustering of space elements $C_{0}$ results in clusters $C_{1}$ and $C_{2}$ which may be equated to virtual particles. Clusters of virtual particles result in $C_{3}$, for example a plasma or the interaction object (see Section 4.4 ). $C_{3}$ clusters are confined in a limited area of curved space. If the area of space expands and the confinement is lost, the virtual particles (i.e., the $C_{1}$ and $C_{2}$ clusters) become real particles. ${ }^{8}$ The real particles may cluster to quantum objects.

De-clustering occurs either (1) as result of "lost fluctuations" or (2) as the result of space expansion. "Lost fluctuations" are fluctuations that do not return to the source cluster because they merge with fluctuations from other sources.

Clustering occurs if (1) the two joining components have compatible types and (2) there exists a suitable coupling force, and (3) the distance between the components is below a certain limit.

Table 1. Hierarchy of clusters of space elements.

\begin{tabular}{|c|c|c|}
\hline Object type & Properties, Attributes & Examples \\
\hline$C_{0}$ (basic) Space Element & curvature, connections, type & simplexes of CDT \\
\hline $\begin{array}{l}C_{1} \text { - Cluster of } C_{0} \text { of same type } \& \\
\text { momentum }\end{array}$ & mass, spin, fluctuating? & virtual particles \\
\hline $\begin{array}{l}C_{2} \text { - Cluster of } C_{1} \text { of differing } \\
\text { momentum }\end{array}$ & mass, spin, fluctuating & virtual particles \\
\hline $\begin{array}{l}C_{3} \text { - Cluster of } C_{1}, C_{2} \text { of differing } \\
\text { types }\end{array}$ & fluctuating & $\begin{array}{c}\text { Plasma, Interaction Object initial } \\
\text { Big Bang, Black Hole? }\end{array}$ \\
\hline $\begin{array}{l}C_{R} \text { - Cluster of } C_{0} \text { of same type } \\
\text { differing momentum }\end{array}$ & mass, spin, fluctuating & real particles \\
\hline Quantum Object & $\begin{array}{l}\text { fluctuating, global attributes, } \\
\text { multiple paths }\end{array}$ & $\begin{array}{l}\text { real particles, collection of real } \\
\text { particles }\end{array}$ \\
\hline
\end{tabular}

${ }^{8}$ This process is similar to the process described in [13] for the evolution of the very early universe according to the advanced Big Bang theory. 


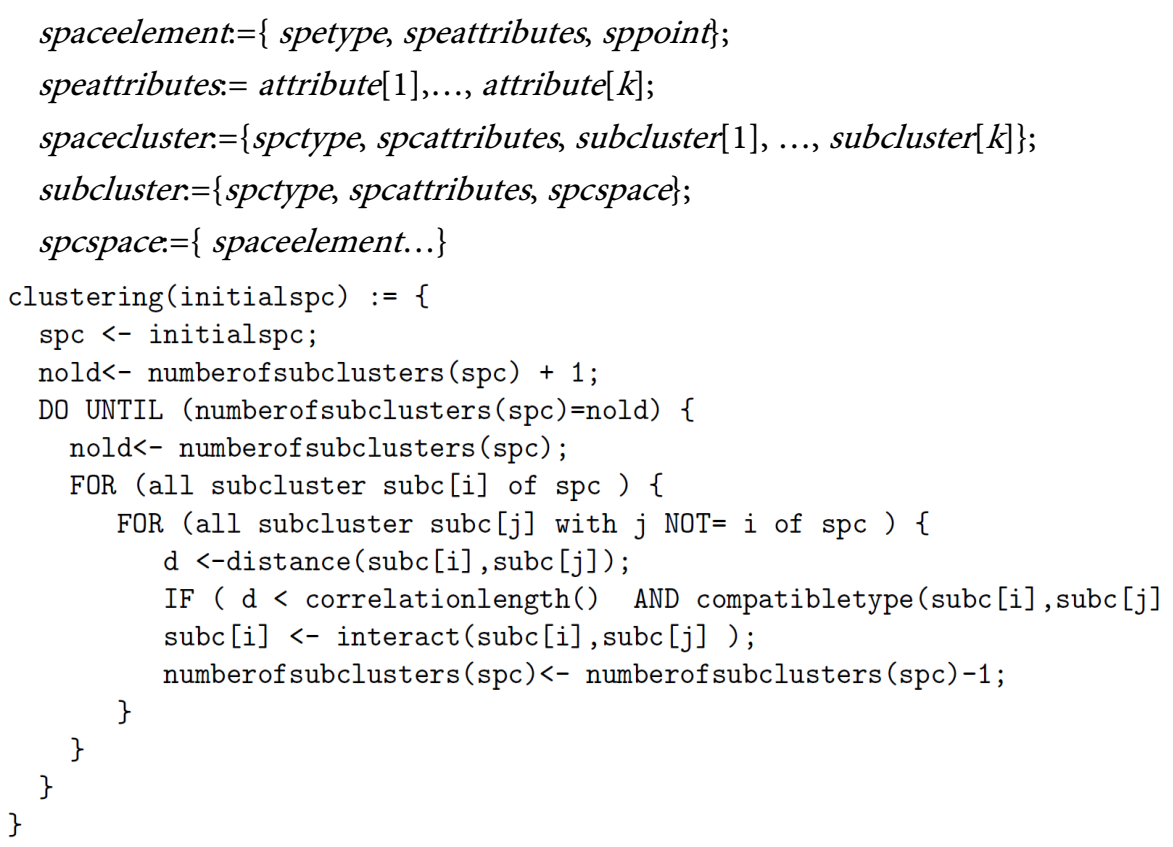

Within the above specification of the clustering process, three areas that are only roughly (or not at all) specified are essential for a concrete clustering process (as, for example, the one described in Section 4.4):

1) With a given concrete clustering process the ultimate development depends also on the movements of the subclusters between the repetitive clustering steps. In the above specification this movement is ignored.

2) The "correlationlength()" determines not only the minimal distance below which an interaction (i.e., clustering) can occur. It also implies that clustering is not restricted to immediate neighbors subc[i], subc[j]. All subclusters within the distance $<$ correlationlength() are subject to clustering. In addition, "correlationlength()" indicates that it is not necessarily a constant, but may be a function that depends on certain parameters such as, for example, the size of the interacting subclusters.

3) "interact(subc[i], subc[j])" is the most important area where concretion is required. For clustering, "merge()" can be a substitute for interact(). For merge(), it must be specified which parts of the sub-clusters subc[i] and subc[j] are to be merged and what the type and attributes must be.

In addition to the local effect associated with the individual interact(), it is important to notice the overall (i.e., global) effect that is associated with the overall clustering. For the clustering described in this article (in particular in Section 4.4), it is assumed that within the cluster, actions propagate instantaneously, at least within the reach of the correlationlength(). Whether the instantaneous action propagation applies to the complete cluster is another detail that must be determined with the concrete clustering case. In [11], Kogut discusses the "correlation length scaling hypothesis" in the context of LGT. The correlation length scaling hypothesis claims that at a critical point (i.e., critical region) "the system is no longer sensitive to the lattice and its small spacing". For the 
causal model of QT/QFT, this may be interpreted as the emergence of a new quantum object with object-global (i.e., cluster-global) attributes. The QFT interaction process described in Section 4.4 includes several examples of clustering and declustering. For the clustering that occurs in QFT interactions, the concretions requested above must be specified such that the results of QFT interactions are in accordance with the predictions of QFT.

Quantum objects: The quantum object is the most important entity for the description of the causal model of QT/QFT. A particle may occur as a separate quantum object or be part of a quantum object. The following three properties distinguish quantum objects from other objects that typically occur in physics:

1) Quantum objects are composed of multiple alternative paths with associated probability amplitudes. As the result of interactions (including measurements), the multiple paths may be reduced to a single path.

2) Quantum objects may consist of multiple spatially separated particles.

3) Quantum objects have global attributes that apply to all of the paths and particles of the quantum object.

The combination of these three properties make quantum objects special within physics.

A quantum object may be viewed as having a two-dimensional structure. One of the dimensions represents the collection of quantum object elements, which typically consists of 1 to $n$ particles.

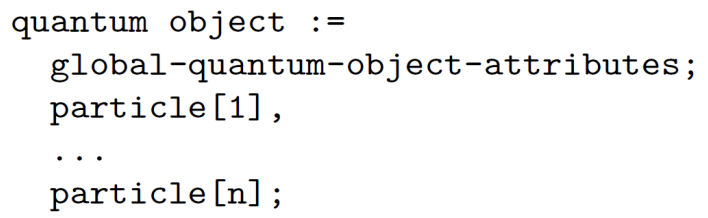

In the second dimension, the quantum object consists of the set of alternatives that may be selected during the evolution of the quantum object, for example, by a measurement.

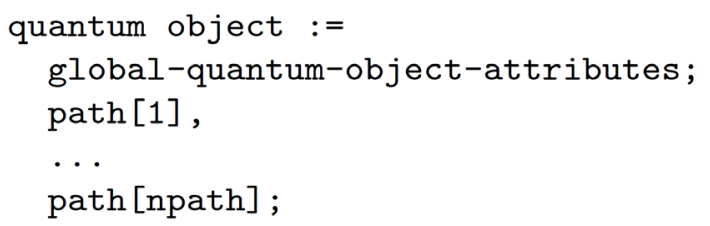

The two-dimensional structure is supplemented by global attributes.

Different types of quantum objects can be distinguished:

- A single particle constitutes the simplest type of quantum object.

- Collections of particles that can be described by a common wave function for which only specific attribute combinations can occur as measurement results represent quantum objects. Thus, the particle collection is represented by a set of paths, and each path contains the attribute combinations for all of the particles and associated probability amplitudes.

- Composite objects such as hadrons, nuclei, and atoms that are built from (elementary) particles are incorporated into the concept of a quantum object. 
- In [7], further types of quantum objects are described that are created during the processing of QFT interactions.

\subsection{QFT Interactions}

Interactions between quantum objects are the key for the causal model of QT/QFT “problem areas” (the QT measurement problem, the QT "interference collapse rule" and entanglement (see [3])). Interactions are also key for the collective behavior processes described in Section 4. In the following, the discussion is restricted to the interactions between two particles. In QFT, such interactions are considered scatterings and they are treated by QFT concepts such as a scattering matrix, Feynman rules and Feynman diagrams.

The collective behavior interpretation of QFT interactions is largely represented in the collective clustering/declustering of the components that constitute the interacting particles. The overall scenario is as follows:

- The interaction between the two particles starts with the interactions between the fluctuations issued by the two particles.

- The interactions between the fluctuations result in (1) the formation of new space element clusters and (2) the declustering of the interacting particles due to lost fluctuations (see Section 4.3).

- When the new space element clusters reach a certain state, they transit into a quantum object which in [6] is called an "interaction object".

- When the declustering of the interacting particles gets below a certain measure, they no longer represent a quantum object. They no longer participate in interactions with other quantum objects, i.e., they are no longer observable. This is then called "the collapse of the wave function".

- The attributes and properties of the generated new interaction object depend on those fluctuations of the interacting particles that initiated the interaction. (This rule is important for the explanation of the measurement process (see below)).

The declustering of the interacting particles and the formation of a new cluster, called an interaction object, is illustrated in Figure 1.

particle-1

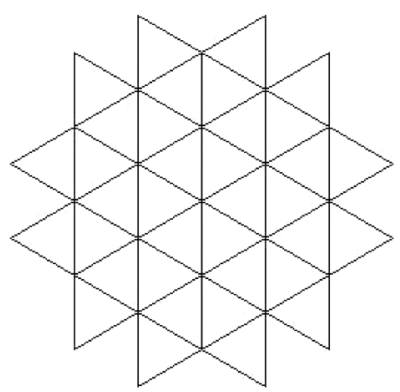

space element cluster-1 cloud

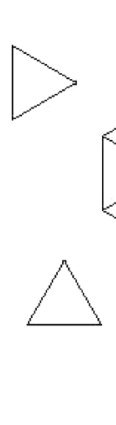

emerging new space element cluster particle-2

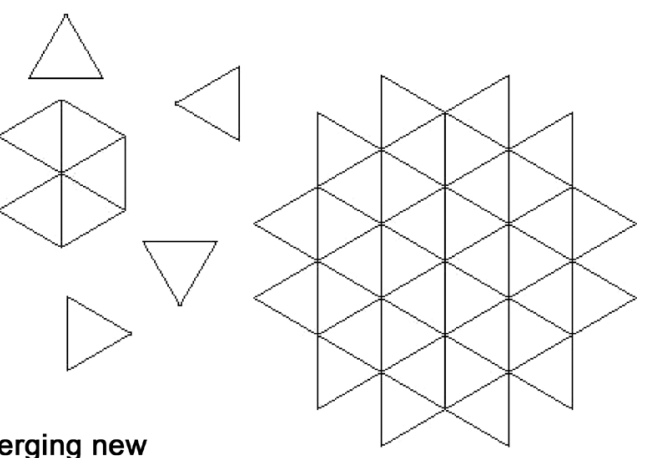

space element cluster-2

Figure 1. Clustering and De-clustering with QFT interactions. 


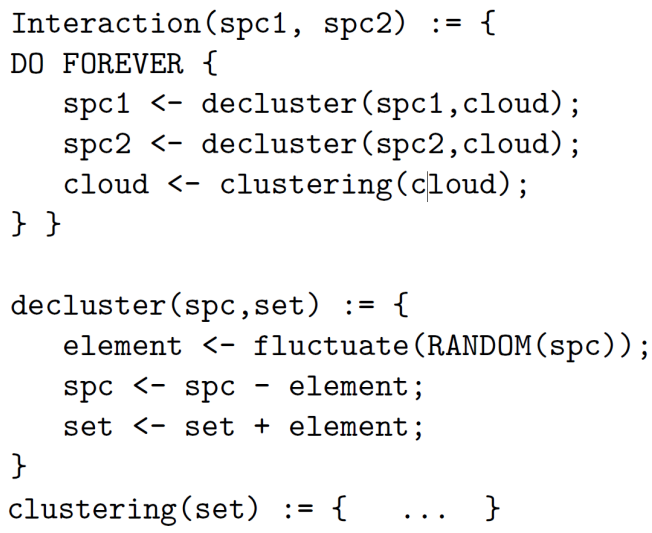

In Section 4.3, clustering() is specified in a generalized form. The items that require a case-specific concretion are discussed in the following. The first concretion is that "sub-clusters" are equated to "virtual particles".

1) The assumption that the virtual particles move during the performance of the clustering process appears to be a plausible assumption. In QFT, to which equivalence must be achieved, the basis for the model of the movements is given by the Feynman diagrams. QFT computation of the scattering matrix can be performed in position space or in momentum space. Considering the movements of virtual particles in space, position space computation appears to be the suitable base for the clustering process. With a closer look, however, it turns out that there are severe inhibitors to using QFT position space computation or momentum space computation as a base for a model of sub-cluster movement during the clustering process. In addition, the causal model of space-time dynamics described in Section 4.2 assumes that space curvature already originates from the lowest level of quantum object, i.e., from the interaction object. This complicates the matter even further. In summary, the author is (at present) not capable of presenting a convincing model for the movement of the virtual particles that constitute the interaction object. However, this is not an unusual situation with collective behavior processes. Still, it appears reasonable to assume that there exists sufficient movement to let the clustering process continue until the maximal cluster(s) are obtained.

2) The "correlationlength()" is another item that needs concretion with QFT interactions. In the original standard QFT, there is no parameter such as correlationlength. An interaction between two waves $\psi_{1}$ and $\psi_{2}$ resulting in a third wave $\psi_{3}$ is described by an equation of motion in which the product of waves $\psi_{1}$ and $\psi_{2}$ is related to $\psi_{3}$; as, for example, in $\mathrm{d}^{2} \psi_{3} / \mathrm{d} t^{2}-c^{2} \mathrm{~d}^{2} \psi_{3} / \mathrm{d} x^{2}=a^{2} \psi_{3}+b \cdot \psi_{1} \psi_{2} \quad$ An interaction occurs if, for a specific position $x_{0}$ the product $\psi_{1}\left(x_{0}, t\right) \cdot \psi_{2}\left(x_{0}, t\right)$ becomes non-zero, which means that both $\psi_{1}$ and $\psi_{2}$ must be non-zero at this position (see, for example [14]). In lattice gauge theory (LGT), correlation length and correlation function are important parameters. In [11], the correlation length $\xi(T)$ "gives the measure of the size of patches of correlated spins in the system". However, as J. Kogut writes in [11] for LGT, "the lattice is mere scaffolding-an intermediate 
step used to analyze a difficult nonlinear system of an infinite number of degrees of freedom." In the causal model of QT/QFT, the assumption of discretization and of a variable (non-zero) correlation length is not just scaffolding. These features have physical implications. As described in Section 4.3, the correlation length also specifies the distance within which the instantaneous action propagation may occur within a cluster. This may be interpreted as the implementation of object-global properties.

3) "interact(subc[i],subc[j])": In QFT, the interaction is expressed in terms of creation and annihilation operators. For quantum electrodynamics (QED) the operator equation has the form (see [15], page 111)

$$
H_{W}(x)=-e N\left\{\left(\bar{\psi}^{+}+\bar{\psi}^{-}\right)\left(/ A^{+}+/ A^{-}\right)\left(\psi^{+}-\psi^{-}\right)\right\}_{x}
$$

where $\bar{\psi}^{+}, \bar{\psi}^{-}, / A^{+}, / A^{-}, \psi^{+}, \psi^{-}$are the creation and annihilation operators. The Equation (1) comprises four variants of photon absorption, $e^{-}+\gamma \rightarrow e^{-}$, $e^{+}+\gamma \rightarrow e^{+}, e^{-}+e^{+}+\gamma, \gamma \rightarrow e^{-}+e^{+}$and four variants of photon emission, $e^{-} \rightarrow e^{-}+\gamma, e^{+} \rightarrow e^{+}+\gamma, e^{-}+e^{+} \rightarrow \gamma, \rightarrow e^{-}+e^{+}+\gamma$. These cases also represent the basic building blocks for the construction of the Feynman diagrams for a given scattering example. For the clustering scenario, the eight cases show the possible combinations of sub-cluster types and the resulting merged (sub-) cluster. In addition, it is assumed that the merge of two sub-clusters of equal types is also possible (this may be viewed as interference).

\section{Collective Behavior Processes as Possible Explanations for Part of the QT/QFT Non-Localities}

In the following, those processes of the causal model of QT/QFT are described in which a refinement by the application of the concepts and techniques of collective behavior appears feasible. In particular, the application of collective behavior concepts should result in a refined explanation of the non-spacepoint-localities within these processes.

\subsection{Measurements in QT}

Measurements in QT refers to that part of the causal model that specifies the transition from probabilities to facts. It is an indispensable assumption for the causal model of QT/QFT that measurements (including the peculiarities of the measurement problem) must be explained by "normal" QT/QFT causal model functions, i.e., by functions that do not refer to concepts such as "measurement" or related terms such as "observer" or "observable". The causal model of QT/QFT described in [14] provides such an interpretation of QT measurements.

In the model described in [16], the key assumption is that a measurement in QT must contain at least one QFT interaction of the kind described above (Section 4.4), i.e., an interaction that contains the "collapse of the wave function". As described above, the QFT interaction causes the formation of an "interaction object" and the attributes and properties of the interaction object depend on 
those parts of the interacting particles that triggered the interaction. This means that the measurement result (which is represented in the interaction object) reflects only part of the information about the state of the measured quantum object. The QFT interaction does not provide a bijective mapping of the state of the measured quantum object to the interaction object. The further peculiarities of the QT measurement are also a consequence of this non-bijective mapping.

\subsection{The Collapse of the Wave Function}

The collapse of the wave function, i.e., the reduction of the set of alternative measurement results to a single definite measurement result, is a key ingredient of the causal model of the QT measurement process and of QFT interactions in general. No matter what the overall assumption of the "interpretation" of QT is, there is apparently agreement among QT physicists that some action occurs that instantaneously affects the complete spatially distributed representation of the particle. In the causal model described in [6] and [7], this non-local action has been explained by the (instantaneous) change of an object-global property. This explanation is refined here by the collective declustering of the components that constitute the particle. Further details have been given above (Section 4.4), where the declustering (i.e., collapse) of the interacting particles is described as the result of lost fluctuations.

\subsection{The Collapse of Interference}

The collapse of interference refers to the process associated with the double-slit experiment when the interference caused by the two slits disappears because of some action at one of the slits such that the path taken can be determined. In [17], the author presented an explanation of the collapse of interference associated with the double-slit experiment based on the causal model of QT/QFT. The explanation presented is the same as the explanation given for the collapse of the wave function, namely, that the occurrence of a QFT interaction causes part of the wave (function) to be abandoned. The more detailed scenario is identical to the one described above for the collapse of the wave function.

\subsection{Entanglement}

Entanglement is the phenomenon that caused the discussion of the feasibility of local causal models of QT. This paragraph considers the related measurement process, i.e., the process that uncovers the (apparent) non-local causality. Above (see Section 5.1), the QT measurement is described as consisting of normal QFT interactions. This is valid also for the two measurements required to measure entangled particles. In addition, for the measurement of entangled particles it must be explained how the measurement at particle- 1 can influence the result of the measurement at particle-2, especially when the two measurements are performed at distant locations. The explanation given in [16] assumes that the two entangled particles belong to a common single quantum object with common 
alternative paths for both particles. The first measurement already reduces the set of paths to a single path such that the second measurement can deliver only the correlated value. Although the measurement of a non-entangled particle and the implied collapse of the wave function can be explained by collective behavior (i.e., declustering), a similar collective behavior interpretation has not yet been found by the author for the collapse of the common wave function of two entangled particles.

\section{5. (De-) Coherence as a Collective Behavior Phenomenon?}

In the causal model of QT/QFT described in [6] and [7] and refined above, the QT measurement process is explained by the collapse of the wave function due to the interaction(s) between the quantum object being measured and the measurement apparatus. In an alternative "interpretation" of QT that is supported by many physicists the QT measurement process is explained by the decoherence of the wave (see [18]). Because the decoherence theory alone can give only an incomplete explanation of the measurement process, the decoherence interpretation is usually combined with Everetts many-worlds interpretation (see [19]). For various reasons, the author does not see the many-worlds interpretation as suited for inclusion into a causal model of QT/QFT. However, if along the lines described Sections 4 and 5, the decoherence theory were refined towards discrete collections of space-time elements (as opposed to continuous waves), this would eliminate the need for the supplementation by the many-worlds interpretation.

If decoherence can be explained as a collective behavior phenomenon, this does imply that coherence can also be understood as a collective behavior phenomenon. If decoherence can be explained as a collective behavior phenomenon, "declustering" may be a better name for it.

\section{Discussions}

\subsection{Do Local Laws Follow from Global and Collective Laws or Vice Versa?}

Because of the broad range of applicability of the laws of collective behavior to different areas of physics, experts in this field (see, for example [11] [8] [20]) suspect that the laws of collective behavior might be the more general laws of physics and that the laws of a single (or a small number of) object(s) may follow from the laws of collective behavior. The laws of collective behavior would represent a kind of high level laws from which the low-level laws of special areas would follow. This view of the relationship between global laws and local laws is not supported by the analysis presented in this article. The laws of collective behavior have a broader range of applicability, but the large applicability is achieved by abstractions of the low-level laws. Thus, it would be equally valid to say that the laws of collective behavior have been derived from the low-level laws (especially the laws of interacting components). 
This does not diminish the value and importance of the laws of collective behavior for various areas of physics. As the most famous example, lattice gauge theory applied the laws of collective behavior to obtain an improved understanding of quantum chromodynamics (see [10] and [11]). Another example of an area of physics in which the application of the laws and techniques of collective behavior resulted in an improved understanding is the refined Big Bang theory that has been developed during the recent decades (see, for example [13]). As a further example, Section 5 describes the refinement of still-weak areas in the (local) causal model of QT/QFT by use of the laws and techniques of collective behavior.

\subsection{Is the End of Reductionism Reached?}

In discussions about collective behavior sometimes "the end of reductionism" is propagated. Usually, it is not described which kind of reduction is meant in this context. Different types of reduction are imaginable:

- reduction towards finer units of space, time, etc.;

- reduction towards more detailed (local) causal relationships;

- reduction towards a smaller number of general laws of physics from which todays laws of physics can be derived (i.e., a theory of everything).

The author believes that with all three types of reduction an end is imaginable but in differing time frames. With none of the three types of reduction does the author see a connection to the growing importance of laws of collective behavior.

In addition, an end of reductionism (with any of the three aspects) would not at all mean that everything is understood in physics. Ironically, the application of the laws and concepts of collective behavior to the refinement of certain theories (e.g., quantum chromodynamics, Big Bang theory, causal model of QT/ QFT) demonstrates that the great potential value of collective behavior may exist with refinements at the micro level of physics as opposed to the finding of general global laws. In other words, collective behavior concepts may enable another step in reductionism (rather than terminate reductionism).

\section{Conclusions}

The causal-model-based analysis of collective behavior presented in this article does not support the vision expressed by some experts in collective behavior that the laws of collective behavior may be the more general, i.e., high-level laws from which the area-specific (low-level) laws follow. Nor does the analysis support the presumption that the findings in connection with collective behavior indicate the end of reductionism. ${ }^{9}$

This does not diminish the value and importance of the laws of collective behavior for various areas of physics. The analysis presented in this article does support the claim that the laws of collective behavior are very important and can

${ }^{9}$ The end of reductionism may or may not be reached for other reasons. 
be applied to many areas of physics to achieve an improved understanding. Examples of such areas have been presented (lattice gauge theory, refined Big Bang theory), including a refinement of the causal model of QT/QFT proposed by the author in [6] [7] and [21]. The improvements in the causal model of QT/QFT affect non-localities in QT/QFT that impeded the construction of a local causal model. The finding that the concepts and laws of collective behavior may help to remove obstacles to the construction of local causal models (especially with the treatment of non-localities) was a surprise even to the author.

The possible reduction of the non-localities in QT/QFT (and in the proposed causal model of QT/QFT) to collective behavior (see Section 5) requires further refinements with respect to the basic elements of collective behavior and the clustering processes. This necessitates a deepening of the model of curved discrete spacetime that have been roughly described in Section 4.2.

\section{References}

[1] Bell, J.S. (1987) On the Einstein-Podolsky-Rosen Paradox. In: Speakable and Unspeakable in Quantum Mechanics, Cambridge University Press, Cambridge.

[2] Aspect, A., Dalibard, J. and Roger, G. (1982) Experimental Tests of Bell's Inequalities using Time-Varying Analyzers. Physical Review Letters, 49, 1804-1807. https://doi.org/10.1103/PhysRevLett.49.1804

[3] Diel, H. (2016) The Completeness, Computability, and Extensibility of Quantum Theory.

[4] Wolfram, S. (2002) A New Kind of Science. Wofram Media.

[5] Ilachinski, A. (2001) Cellular Automata: A Discrete Universe. World Scientific. https://doi.org/10.1142/4702

[6] Diel, H. (2016) Are Local Causal Models of Quantum Theory Feasible at All?

[7] Diel, H. (2016) Quantum Objects as Elementary Units of Causality and Locality.

[8] Satz, H. (2013) Gottes unsichtbare Würfel. Verlag C.H. Beck, München. https://doi.org/10.17104/9783406655500

[9] Wegner, F. (1971) Duality in Generalized Ising Models and Phase Transitions without Local Order Parameters. Journal of Mathematical Physics, 12, 2259-2272. https://doi.org/10.1063/1.1665530

[10] Wilson, K.G. (1974) Confinement of Quarks. Physical Review D, 14, 2455.

[11] Kogut, J.B. (1979) An Introduction to Lattice Gauge Theory and Spin Systems. Reviews of Modern Physics, 51, 659-714. https://doi.org/10.1103/RevModPhys.51.659

[12] Loll, R., Ambjorn, J. and Jurkiewicz, J. (2005) The Universe from Scratch.

[13] Satz, H. (2016) Kosmische Dämmerung. Verlag C.H. Beck, München. https://doi.org/10.17104/9783406697883

[14] Strassler, M. (2017) Conversations about Science with Theoretical Physicist. https://profmattstrassler.com/articles-and-posts/particle-physics-basics/

[15] Mandl, F. and Shaw, G. (1993) Quantenfeldtheorie. AULA Verlag, 351.

[16] Diel, H. (2014) A Model of the Measurement Process in Quantum Theory.

[17] Diel, H. (2015) An Improved "Interference Collapse Rule" of Quantum Mechanics. Open Access Library Journal, 2, e1838. https://doi.org/10.4236/oalib.1101838

[18] Schlosshauer, M. (2007) Decoherence and the Quantum-to-Classical Transition. 
Springer Verlag, Berlin.

[19] Everett III, H. (1957) Relative State' Formulation of Quantum Mechanics. Reviews of Modern Physics, 29, 454. https://doi.org/10.1103/RevModPhys.29.454

[20] Laughlin, R.B. (2006) A Different Universe: Reinventing Physics from the Bottom Down. Basic Books.

[21] Diel, H.H. (2017) Spacetime Structures in a Causal Model of Quantum Theory. Open Access Library Journal, 4, e3357. https://doi.org/10.4236/oalib.1103357

Submit or recommend next manuscript to OALib Journal and we will provide best service for you:

- Publication frequency: Monthly

- 9 subject areas of science, technology and medicine

- Fair and rigorous peer-review system

- Fast publication process

- Article promotion in various social networking sites (LinkedIn, Facebook, Twitter, etc.)

- Maximum dissemination of your research work

Submit Your Paper Online: Click Here to Submit

Or Contact service@oalib.com 